\title{
Anabases
}

ANABASES Traditions et réceptions de l'Antiquité

12 | 2010

Varia

\section{Cirillo e Ipazia nella storiografia cattolica}

\section{Luciano Canfora}

\section{(2) OpenEdition}

\section{Journals}

Edizione digitale

URL: http://journals.openedition.org/anabases/1111

DOI: 10.4000/anabases. 1111

ISSN: 2256-9421

\section{Editore}

E.R.A.S.M.E.

\section{Edizione cartacea}

Data di pubblicazione: 1 ottobre 2010

Paginazione: 93-102

ISSN: 1774-4296

\section{Notizia bibliografica digitale}

Luciano Canfora, «Cirillo e Ipazia nella storiografia cattolica », Anabases [Online], 12 | 2010, Messo

online il 01 octobre 2013, consultato il 20 octobre 2019. URL : http://journals.openedition.org/ anabases/1111 ; DOI : 10.4000/anabases.1111 
Anabases 12 (2010), p. 93-102.

\section{Cirillo e Ipazia nella storiografia cattolica}

LUCIANO CANFora

LA VICENDA DI IPAZIA è stata largamente studiata. Ottimi lavori sono stati prodotti nel tempo. Il suo nome è stato difeso anche nei momenti di maggiore prevalenza dell'oscurantismo clericale. Anche la storia della sua fama ha avuto adeguato rilievo. Merita dunque attenzione, perché rischia di passare inosservato, il modo falsificante con cui strumenti di studi programmaticamente cattolici (e perciò consapevolmente di parte) riferiscono i fatti.

1. Il tema è certo imbarazzante. Sono di fronte: una scienziata alessandrina trucidata dai monaci cristiani perché non incline a conversioni di comodo ed un vescovo molto potente e ormai soverchiante rispetto al potere statale che si fa mandante morale della uccisione, plateale e sadicamente feroce, di una donna, che è anche una notevole scienziata, colpevole di non voler essere cristiana ma assertrice della filosofia e della scienza greca. Epilogo: quel vescovo, così politico e così spregiudicato (per non dir peggio) viene - post mortem - proclamato santo e «dottore della chiesa» (per aver escogitato lo stravagante dogma che proclamò Maria «madre di Dio», $\theta \in$ Єо́коร, in quanto madre di Gesù, cioè del «figlio»).

La fonte che più disturba gli studiosi cattolici è Socrate, storico ecclesiastico coevo dei fatti, cristiano di simpatie novaziane e autore di una Storia ecclesiastica nel cui VII libro la vicenda di Ipazia è trattata con molta onestà (capp. 13-15). Il racconto di Socrate chiama in causa apertamente Cirillo, ed è quanto mai limpido. Socrate dice molto chiaramente che la liquidazione fisica di Ipazia fu, per Cirillo, lo strumento mirante a far accettare alla parte meno violenta della comunità cristiana di Alessandria la beatificazione di un monaco brutale e teppistico, tale Ammonio, che il prefetto 
Oreste, vittima di una sua aggressione, aveva fatto giustiziare. Cirillo tentò subito di fare adorare Ammonio come martire col nome di Taumasio. Ma - precisa Socrate «le persone equilibrate (oi $\sigma \omega \phi \rho \circ \nu$ oṽ $T \epsilon \zeta$ ), quantunque cristiane, non approvavano tale eccessivo zelo di Cirillo nei confronti di costui» (VII, 14). In una tale impasse l'aggressione a Ipazia, nota come amica di Oreste, gli fu preziosa. E qui Socrate fornisce i dettagli dell'aggressione e del martirio di Ipazia (VII, 15) ad opera di una squadra di

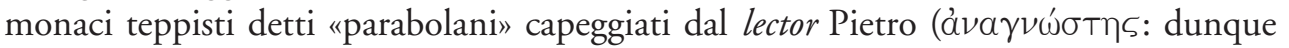
non un analfabeta) che costituivano, per Cirillo, una specie di "guardia del corpo ${ }^{1} »$ : catturata per la strada, denudata, lapidata, fatta a pezzi e bruciata. Commenta Socrate, al termine di quel capitolo: "Questo misfatto procurò non poco biasimo (oủ $\mu$ นкpòv $\mu(\tilde{\mu} \mu \nu)$ a Cirillo e alla chiesa di Alessandria.» La parola usata ( $\mu \tilde{\omega} \mu \mathrm{s})$ indica biasimo, disonore, macchia. Va da sé che, solo in quanto considerato mandante o ispiratore, Cirillo poté essere oggetto di "grande biasimo», di "macchia», anche perché non risulta abbia fatto alcunché per condannare l'accaduto. La sua complicità è affermata anche da Damascio, che leggiamo negli estratti compresi nella voce Ipazia del lessico bizantino di Suidas ( $(, 166)$. Damascio è il neoplatonico autore della Vita di Isidoro, che vive un secolo più tardi. Socrate è cristiano e coevo dei fatti: le sue date approssimative sono 370-430 d.C.

Henry Dominique Saffrey ha ricostruito bene l'intera vicenda. Essa prende le mosse dall'attacco distruttivo contro gli Ebrei di Alessandria ad opera dei 500 monacisquadristi fatti giungere in città dalla Nitria su ordine di Cirillo che in Nitria tra quei monaci aveva a lungo soggiornato. Oreste ha protestato contro tale pogrom presso la corte a Bisanzio, e perciò è stato bersagliato dalle violenze di Ammonio e dei suoi monaci sfociate poi nel massacro di Ipazia: «Cyrille ne pouvant atteindre directement son adversaire, ses partisans dirigèrent leur hostilité sur un personnage de l'entourage du préfet, ce fut Hypatie [...] sans doute à l'instigation du patriarche qui rétablit du coup son autorité ${ }^{2} . »$

2. Come liberarsi della testimonianza di Socrate? La via più «classica» e tradizionale consiste nello squalificare la fonte. È la strada scelta dall'Enciclopedia cattolica nella voce Cirillo d'Alessandria ${ }^{3}$ : «Se bisogna credere a Socrate gli inizi del suo [di Cirillo] episcopato furono segnati da continue rivolte e violenze in cui restò compromesso indirettamente il vescovo stesso [...]. La causa principale di queste agitazioni fu la

1 «Die Schutztruppe des Bischofs» scrive efficacemente Henriette Harich nella sua tesi dottorale Hypatia, Graz, 1997, p. 127.

2 H.D. SAFFreY, Dictionnaire des philosophes antiques, IV, éd. CNRS, Paris, 2005, s.v. Hypatie d'Alexandrie, p. 817, che rinvia anche a P. CHUvin, Chronique des derniers païens, Belles Lettres, Paris, 1990, p. 91-94 ed a Chr. HAAS, Alexandria in Late Antiquity. Topography and social conflict, The Johns Hopkins University Press, Baltimore-London, 1997, p. 313-316.

3 Vol. II (1950), col. 1716. La voce è firmata da M. Jugie. 
discordia con il prefetto Oreste. Nel 415 durante una baruffa [sic ! ${ }^{4}$, la celebre Ipazia, versatissima nella filosofia neoplatonica, fu ammazzata da un parabolano a causa della sua presunta influenza presso il prefetto. Non si può imputare a Cirillo questo assassinio nonostante le insinuazioni di Socrate, il quale non è imparziale.»

Ed anche il ben più serio Dictionnaire de Théologie catholique diretto da Émile Amann adotta, quantunque meno rozzamente, la stessa linea: «Tous nos renseignements sur cette première période [412-428 d.C.] nous viennent de l'historien Socrate: le portrait qu'il nous présente est-il parfaitement exact?"

La soluzione adottata dall'autorevole Dizionario ecclesiastico di Angelo Mercati e Augusto Pelzer ${ }^{6}$ è ancora più semplice: falsificare la cronologia di Socrate, spostarlo nel secolo successivo e sentenziare che dunque non poteva sapere perché vissuto un secolo dopo i fatti. Ecco le parole dell'autorevole Dizionario: «[Ipazia] fu uccisa in una dimostrazione popolare [sic!] perché avversa al cristianesimo; non fu però mai provata la colpevolezza di San Cirillo Alessandrino in questo fatto, come pretesero un secolo più tardi il filosofo Damascio nella sua Vita Isidori e lo storico bizantino Socrate nell' Historia ecclesiastica (VII, 15 = PG LXVII, 768)».

$\mathrm{Al}$ di là della falsificazione grossolana, questa pagina è interessante: infatti riconosce che le parole di Socrate dichiarano la colpevolezza di Cirillo. Il che smentisce l'altra "linea difensiva» instaurata da un altro autorevole repertorio cattolico, il Lexikon für Theologie und Kirche dell'editore Herder di Freiburg i. Br., consistente nel sostenere - con involontaria comicità - che invece, a rigore, Socrate non parla di "colpevolezza" ma di «vergogna»: «Dazu [cioè dopo la cacciata degli Ebrei da Alessandria] kommen dunkle Ereignisse: Kampf von Mönchsscharen gegen den Präfekten Orestes, die grausame Tötung der Philosophin Hypatia durch einen vom Lektor Petrus organisierten christlichen Mob. Sokrates [PG LXVII, 769] spricht wohl von der "Schande" für Kyrillos und die Kirche von Alexandrien, nicht aber von einer Mitschuld Kyrillos» (VI, 1961, col. 707) ${ }^{7}$. A sostegno di questa forzata interpretazione delle parole di Socrate, l'articolista del Lexikon invoca una autorità: «W. Jaeger, RGG³ III 502».

Werner Jaeger fu infatti autore, nel 1956, della voce Hypatia nella terza edizione del repertorio teologico protestante "Die Religion in Geschichte und Gegenwart [= RGG]". $\mathrm{E}$ fu condiscendente verso il contesto confessionale in cui si trovava a scrivere, ma non al punto di falsare la realtà delle fonti. Infatti scrisse che della uccisione di Ipazia «la tradizione fa in parte responsabile il vescovo Cirillo» («Für ihren tragischen Tod (415) durch einen fanatisierten christlichen Mob, der sie unter Anführung des Lektors

4 «Baruffa» è la versione casereccia della formula ipocrita adottata dal Grand Dictionnaire del Moréri (s.v. Hypatia, ed. 1747, p. 357): «Elle fut tuée dans une sédition populaire.»

5 Vol. III, s.v. Cyrille d'Alexandrie, redatta da J. Mahé, Letouzey et Ané, Paris, 1938, col. 2477.

6 Vol. II, s.v. Ipazia, UTET, Torino, 1953, p. 465.

7 Voce firmata da A. Spindeler. 
Petros auf der Strasse angriff [...] macht die Überlieferung z.T. Cyrill verantwortlich»). Dunque Jaeger non dice che Socrate non parla della responsabilità di Cirillo, dice il contrario! Dice che «la tradizione attribuisce a Cirillo una parte della responsabilità». Dopo di che si può anche rimanere perplessi dinanzi ad una formula così ipocrita: dire, soprattutto trattandosi di persona di alta responsabilità come un vescovo - e quel vescovo! -, che ebbe «una parte di responsabilità» significa arrampicarsi sugli specchi: e infatti Jaeger soggiunge salomonicamente che «la battaglia (sic: der Streit) intorno alla partecipazione (Anteil) di Cirillo alla vicenda non è andata al di là di congetture (nicht über Vermutungen hinausgekommen)». Si può ancora di più apprezzare l'equilibrismo dello scrivente, ma certo Jaeger non parla qui di Socrate o di Damascio o di Suidas (i quali tutti incolpano Cirillo!), bensì della discussione moderna («der Streit» è ovviamente la discussione tra i moderni) ${ }^{8}$.

Dunque l'articolista del Lexikon cattolico ha voluto trarre dalla sua l'analogo repertorio protestante, e per giunta anche il nome importante di Jaeger, ma ha solo commesso un falso.

Conclusivamente: è molto istruttivo osservare che nella nuova, ormai terza, edizione del medesimo Lexikon (voce Cyrill von Alexandrien) ${ }^{9}$ ogni riferimento alle "gesta» di Cirillo (pogrom antiebraico, contrasto con Oreste, martirio di Ipazia) è scomparso, e si discetta unicamente di teologia (nebbie trinitarie relative al dogma della $\theta \in$ OTÓKOS etc.). Una ben curiosa autocensura.

La trovata dell' «incidente» avvenuto «in una dimostrazione popolare», o addirittura «durante una baruffa», è stata a quanto pare definitivamente accantonata: tra l'altro perché contrasta in modo troppo sfacciato con l'indicazione della fonte: $̇$ $\pi เ T \eta \rho 0 v ̃ \sigma \iota$

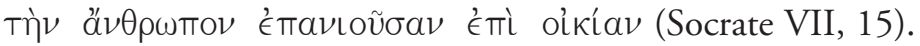

3. Ma nella nobile arte della reticenza che è una forma anch'essa di falsificazione si può essere anche più sofisticati. Nel IV tomo della monumentale Histoire de l'Église diretta da Augustin Fliche e Victor Martin, redatto da De Labriolle, G. Bardy, L. Bréhier e G. De Plinval (Dalla morte di Teodosio all'avvento di Gregorio Magno)

8 Jaeger ha sottomano, mentre scrive questa voce, l'articolo, eccellente, di Karl Praechter della Realencyclopädie (IX, 1916, 242-249) Hypatia, anche se lo "svilisce» indicandone unicamente l'utilità bibliografica ("Weitere Literatur»s. RE IX, $242 \mathrm{ff}$.). Ed invece è di lì che viene il suo salomonico riferimento allo «Streit», giacché l'ultimo e conclusivo capoverso di quell'articolo incomincia proprio così: «Der Streit über die Stellung des Kyrillos zu dem Verbrechen ist in der Neuzeit mit großer Lebhaftigkeit wiederaufgenommen worden. Kulturkampf und Klerikalismus haben sich die H.- Frage zum Kampfplatz ausersehen. Aber der ganze Streit ist müßig, da unsere Akten schlechterdings nicht zureichen, die Frage nach der Beteiligung des Bischofs an der Untat zu bejahen oder zu verneinen» $(248,30-38)$. Nel seguito, peraltro, come vedremo, Praechter indica qual è il sostanziale "contributo" dato da Cirillo alla eliminazione di Ipazia. II, 1994, coll. 1368-1370, voce firmata da Hermann Josef Vogt. 
il racconto di questi eventi procede con quasi fanciullesca semplicità. In tre distinti, brevi paragrafi (La questione di Oreste ${ }^{10}$, Contro gli Ebrei, Contro i Pagani) la vicenda si dipana tra frasi tipo "San Cirillo si trovò impegnato in un penoso litigio con Oreste, prefetto d'Egitto. Non sappiamo per quale motivo...», oppure "Les Juifs eux aussi apprirent à leur dépens à connaître le zèle de Cyrille etc.». Ma il vertice viene raggiunto nel telegrafico paragrafo Contre les païens, che in realtà vuol solo dire "contro Ipazia». Il testo è brevissimo: «À peine est-il besoin d'ajouter que les païens ne furent pas mieux traités. Le meurtre d'Hypatie, en mars 415 , n'est guère qu'un épisode particulièrement douloureux d'une lutte fertile en incidents ${ }^{11}$.»

Lo stesso Bardy, coautore di questo quarto volume, quando scrive «in proprio» la voce Cyrille d'Alexandrie, nel XIII volume del Dictionnaire d'histoire et de géographie ecclésiastiques (1956), varia questa formula in senso più drammatico, e sostituisce al melenso «douloureux» il più colorito «sombre»: «Le meurtre d'Hypatie est l'incident le plus sombre d'un conflit sans cesse renouvelé» (col. 1169).

Non deve sfuggire la complessa manipolazione che questa breve notizia ha sopportato nella edizione italiana del Fliche-Martin, pubblicata a Torino, per cura del prelato A.P. Frutaz, presso l'editrice S.A.I.E. nel 1961. Qui, dopo le parole «fertile en incidents» («ricca di incidenti»), viene interpolata, con opportuni contrassegni che rendono chiara l'operazione, una pagina molto forte di Louis Duchesne (dalla Histoire ancienne de l'Église III, p. 301) che descrive efficacemente e apertamente condanna il massacro di Ipazia:

«Tra le persone più apprezzate dal prefetto Oreste c'era l'illustre Ipazia, donna di elevate facoltà letterarie, stimata per i suoi costumi come per il suo ingegno. Era ancora pagana e dirigeva la scuola neoplatonica; Oreste non era il solo cristiano a stimarla, l'onorava pure il vescovo Sinesio. La corte di Cirillo ritenevala istigatrice di tutti i malevoli progetti prefettizi e responsabile dell'ostilità di Oreste contro il Vescovo. Un giorno, alcuni fanatici, guidati da uno dei lettori di Cirillo, di nome Pietro, l'attesero per via, la strapparono giù dal suo carro, la trascinarono nella chiesa del Caesareum, e là, la spogliarono delle vesti, l'uccisero a colpi di tegola, tagliandone poi il cadavere a pezzi, bruciati in un'orgia di cannibali.»

A questa dura ed efficace ricostruzione però viene aggiunta una nota (p. 195, nota $20-a$ ), ricavata ugualmente dal Duchesne, che, dopo aver ricordato che Socrate è la nostra sola fonte (il che non è esatto: si veda la voce 'Үratía in Suidas), ne dovrebbe limitare il valore: Socrate si sarebbe basato sulle "voci che correvano allora a Costantinopoli, e possono quindi presentare una certa dose di esagerazione». Nell'edizione originale del Fliche e Martin la pagina di Duchesne non c'è (ovviamente), ma ci sono unicamente queste parole tratte dalla nota! In tal modo la posizione

10 L'affaire d'Oreste: titolo di una ipocrisia così pronunciata che travalica nel comico.

11 Histoire de l'Église, IV, Bloud \& Say, Paris, 1948, p. 157. 
di Duchesne ne usciva del tutto falsata. Infatti il testo completo della nota di Duchesne è tutt'altro: «Les histoires ci-dessus nous sont racontées par Socrate. Elles représentent les bruits accrédités à Constantinople et comportent ainsi une certaine dose d'exagération. Toutefois l'impression qui s'en dégage ne saurait être négligée, car elle ne concorde que trop avec ce que d'autres documents et des moins discutables, nous apprennent sur le caractère et les procédés du terrible archevêque» (Histoire ancienne de l'Église III, Paris 1910, p. 301, n. 1).

Eliminata la seconda parte della nota, il senso del pensiero di Duchesne risulta completamente stravolto. Il suo esatto pensiero è invece che, nonostante Socrate si sia dovuto (ovviamente!) basare sulla relazione dei fatti giunta a Costantinopoli (cioè in ultima analisi quella ufficiale del prefetto) e dunque l'ipotesi di qualche esagerazione («une certaine dose!») non sia da escludere - esagerazione evidentemente nei dettagli sulla esecuzione $^{12}$-, il racconto è da ritenersi più che credibile in quanto «si accorda fin troppo con ciò che altri documenti, e dei più indiscutibili, ci dicono intorno al carattere e al modo di agire del terribile vescovo ${ }^{13}$ ”.

Il pensiero di Duchesne viene dunque sconciato. Egli viene costretto ad apparire scettico sulla qualità della testimonianza di Socrate, laddove Duchesne ha innanzi tutto dato rilievo alla ferocia («un'orgia da cannibali»!) dei monaci parabolani, e ribadito nella nota (se letta per intero) che quelli erano i metodi di Cirillo (p. 22) ${ }^{14}$. Perfido trattamento inflitto allo storico filo-modernista amico, un tempo, e frequentatore di Alfred Loisy! 15

Un'ultima chiosa su questa penosa operazione. Fliche e Martin (IV, p. 157) hanno ignorato il racconto efficace e inequivocabile di Duchesne e ne hanno ritagliato, dalla nota 1 di pag. 301, solo le parole che potevano essere piegate a fargli dire l'opposto del suo pensiero. Frutaz, nella traduzione italiana dell' Histoire di Fliche e Martin, ha avuto l'onestà di riprodurre la pagina di Duchesne, ma non ha osato pubblicare anche la nota nella sua interezza perché avrebbe reso evidente la manipolazione operata da Fliche e Martin, nell'opera che egli stesso sta presentando al lettore italiano.

12 L'uso di ostraka per scarnificare il corpo della vittima, ad esempio.

13 La volgarità di questo modo di falsare non solo gli antichi testi ma anche la bibliografia moderna si commenta da sé. Essa può essere smascherata soltanto dalla libertà immanente nel metodo filologico.

$14 \mathrm{Ci}$ sarebbe poi anche da osservare che l'espressione «bruits accrédités à Constantinople» non significa affatto che Duchesne intenda che si trattasse di invenzioni. Tra l'altro «bruits» significa sia notizia che voce e non ha necessariamente valore negativo. Si riferisce piuttosto al modo in cui le notizie si erano potute propagare piuttosto che alla loro veridicità. E comunque è la seconda parte della nota, tagliata di netto da Fliche e Martin, che dà pienamente il senso del pensiero di Duchesne su questa vicenda.

15 Nel Lexikon für Theologie und Kirche, (III [1959], col. 593) si arriva a dire: «Duchesne hat nichts gemein mit dem sogenannte französischen Modernismus»! 
4. Va comunque osservato che, in tutta questa vicenda storiografica, la manipolazione principale consiste nell'affermare che Socrate sia l'unica fonte che parli della diretta responsabilità di Cirillo nell'assassinio. Invece è vero il contrario.

La lunga e dotta voce Ymatía di Suidas è invece a tal proposito molto dettagliata: segnala, tra l'altro, la causa del risentimento di Cirillo - la constatazione de visu, da

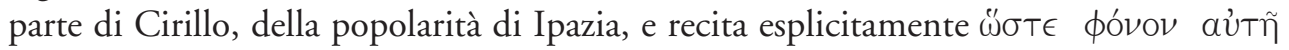

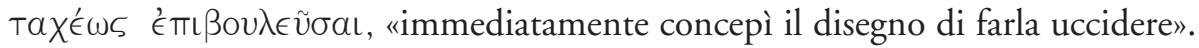

La fonte del lessicografo è Damascio (seconda metà del v secolo), autorevole esponente della Scuola neoplatonica di Atene chiusa da Giustiniano. Argomentare che, vissuto un secolo dopo i fatti, Damascio non poteva sapere è stolto: è come dire che quanto Polibio narra dalla prima guerra punica, rispetto alla quale egli vive per l'appunto un secolo più tardi, non ha valore! (Per non parlare di Tito Livio, che, in base a quel criterio, andrebbe direttamente cestinato).

Non si osa affermare, ovviamente, che Damascio è prevenuto in quanto non cristiano e neoplatonico: bisognerebbe infatti poter dimostrare indipendentemente la sua faziosità e, con procedura comunque azzardata, estendere il giudizio negativo sulla sua affidabilità anche a quanto egli dice di Cirillo. Ma questo non è possibile, e perciò si ripiega sul rilievo «ma è vissuto troppo dopo» implicando che automaticamente ciò comporterebbe che Damascio fosse al contempo disinformato e falsario!

Che la testimonianza di Damascio fosse invece del tutto degna di attenzione e che costituisse una conferma indipendente di quanto narrato da Socrate lo aveva bene inteso Henri de Valois (1604-1676), educato dai gesuiti nel collegio rinomatissimo di Clermont, protégé del cardinale Mazzarino, storico ufficiale di Luigi XIV, editore di rango degli storici ecclesiastici greci (del 1668 è il tomo contenente Socrate). Nel commento al passo di Socrate in cui si parla dell" «onta ricaduta su Cirillo», Valois annota: «Mũ $\mu$ ov Kuṕ́ $\lambda(\omega)$ : certe Damascius in vita Isidori philosophi quam ego duplo auctiorem editurus sum [...] caedis Hypatiae auctorem facit Cyrillum», quindi traduce in un magnifico latino l'intera pagina di Damascio «et cetera quae leguntur apud Suidam», ma non solleva alcun dubbio sulla fondatezza di tale ricostruzione. C’è sempre da esser grati all'indipendenza "gallicana» di una parte della cultura cattolica francese.

5. Una terza fonte, del tutto indipendente, è Filostorgio (368-439 d.C.), ariano di stretta osservanza e fustigatore sia dei pagani che degli atanasiani trionfanti ad Alessandria. È probabile - come ha osservato Joseph Bidez - che Filostorgio, ad Alessandria, abbia ascoltato direttamente le lezioni di Ipazia ${ }^{16}$ : è di Filostorgio l'osservazione secondo cui Ipazia era diventata, nel campo della osservazione degli astri

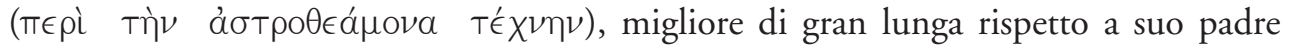
Teone (Hist. eccl. VIII, 9): una notazione che sembra chiaramente dovuta ad esperienza diretta. L'opera di Filostorgio, in quanto di ispirazione ariana, è stata perseguitata e

16 Philostorgius, Kirchengeschichte, ed. J. Bidez, [1913], Berlin $1981^{3}$, p. CIX. 
l'ortodossia «cattolica» ne ha impedito la conservazione. Nondimeno il patriarca Fozio, che per gli eretici aveva uno spiccato interesse, lo ha letto e ne ha dato un riassunto, pur chiamandolo comicamente, ad ogni piè sospinto, «l'empio». Ecco come Fozio riassume il racconto di Filostorgio sull'uccisione di Ipazia: «L'empio a questo punto dice che, al tempo del regno di Teodosio, quella donna fu fatta a pezzi dai sostenitori della

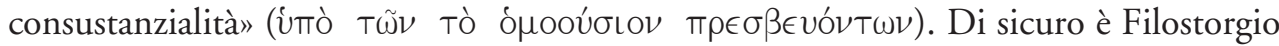
stesso che deve aver usato l'espressione, dal suo punto di vista sprezzante, «i sostenitori della consustanzialità» per riferirsi ai zelanti sostenitori di quella visione teologica - da lui avversata - che, da Atanasio a Teofilo a Cirillo, predominava ad Alessandria. Per Filostorgio dunque l'assassinio non era opera di una amorfa folla fanatica ma di quel clero, capeggiato da Cirillo, che ad Alessandria dominava sia la chiesa che la piazza.

Che poi si trattasse di gente convinta "di fare cosa gradita a Cirillo» (come si esprime l'anonimo autore della voce Ipazia nell' Enciclopedia Italiana vol. XIX [1933], p. 468) o di gente cui Cirillo aveva, allusivamente o esplicitamente, additato quel bersaglio, trattandosi oltre tutto della sua "guardia del corpo", è davvero distinzione irrilevante ${ }^{17}$.

Lo rileva molto efficacemente Karl Praechter nella conclusione della voce Hypatia della Realencyclopädie Pauly-Wissowa (vol. IX [1914], col. 248): «Daß Kyrillos durch seine Schuld oder Mitschuld an dem Zerwürfnis mit Orestes auch zum Untergange Hypatias beigetragen hat, ist nicht zu bezweifeln.»

6. Anche il cronista antiocheno Giovanni Malalas (VI secolo) ${ }^{18}$ conosce una tradizione che indicava Cirillo come mandante dell'assassinio di Ipazia. Scrive Malalas:

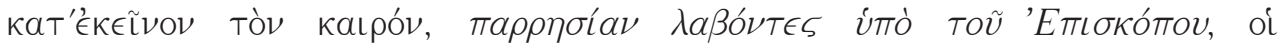

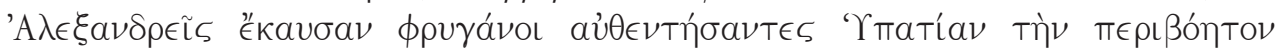

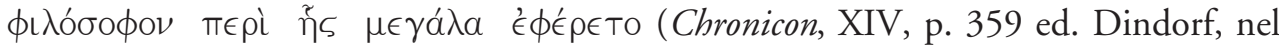
"Corpus Bonnense»). Bene traduce Carlo Pascal l'espressione cruciale di questo passo

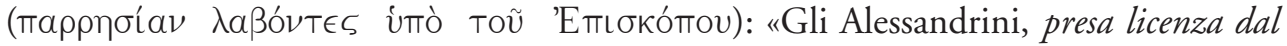
loro vescovo, bruciarono Ipazia con fascine di legna da ardere ${ }^{19}$."

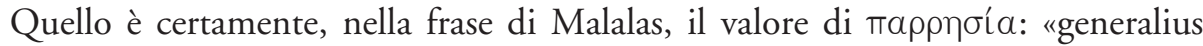
etiam accipi pro Өáppos quidam annotant» segnala il Thesaurus (vol. VII, 556 A). Ed

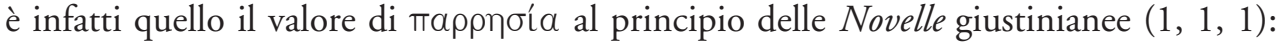
licence, permission traducono correttamente Liddell-Scott-Jones (s.v.).

17 Avvilupparsi in questo genere di distinguo è come ostinarsi a sostenere che Mussolini fu estraneo all'uccisione di Matteotti.

18 La sua cronaca giunge al 563, quasi al termine del regno di Giustiniano.

19 C. PASCAL, Figure e caratteri, Milano, Sandron 1908, p. 172. L'esatto valore di aủ $\theta \in \nu T \epsilon ́ \omega$ è chiarito da Ludwig Dindorf, buon conoscitore di Malalas, nelle aggiunte al Thesaurus Graecae Linguae (vol. II, 2448 A): "Caedem propria manu perpetro», con altri esempi tratti da Malalas. 
L'espressione rende esattamente la dinamica dei fatti: gli oltranzisti, pronti, per la loro fede, anche al crimine, hanno avuto «licenza» da Cirillo. Egli, dopo aver creato il clima adatto, ha fatto ricorso all'ipocrita trovata di lasciar intendere chiaramente che «autorizzava» l'azione dei suoi pretoriani-parabolani, debitamente eccitati dalla sua oratoria.

Sulla storia a lui più vicina, Malalas ha fonti sue proprie, non riconducibili né a Socrate né a Damascio. Siamo dunque in presenza di una vasta e ramificata tradizione che concorda sul punto sostanziale ${ }^{20}$, cioè la grave responsabilità di Cirillo, ma che attinge a distinti resoconti. Malalas, ad esempio, conosce un dettaglio che non passerà

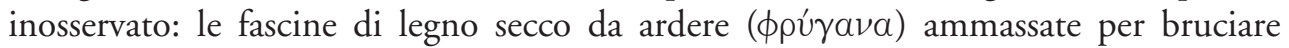

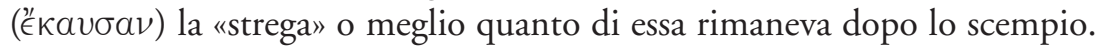

E per concludere sulla questione delle fonti: non si è posto mente ad un elemento fattuale, messo in luce da Henriette Harich nella sua dissertazione, e cioè all'editto, contenuto nel Codex Theodosianus (XVI, 2, 42), con cui l'imperatore ordina la riduzione del numero dei «parabolani», «Schutztruppen» del vescovo. Esso è la risposta, tardiva certo, alle numerose violenze commesse da quelle micidiali squadre con l'avallo di Cirillo.

Il potere politico centrale era lontano: a Bisanzio. Ad Alessandria c'era bensì il praefectus augustalis, ma costui non era in grado di reggere all'urto delle masse cristiane fanatizzate. (Non è un caso che un secolo più tardi Alessandria cadrà, senza seria resistenza, nelle mani dei conquistatori arabi: il progressivo indebolimento del potere politico dava ormai i suoi frutti). Alla luce di tutto ciò si può meglio apprezzare la giusta constatazione di Maurice Croiset a proposito della vicenda di Ipazia: la quale - nota Croiset - fu uccisa «ignominieusement, sans qu'aucune autorité intervînt à temps pour la sauver ${ }^{21}$ ». «Aucune autorité» è molto ben detto, perché non riguarda soltanto l'autorità politica, indebolita e resa impotente, ma soprattutto la vera autorità del momento: il vescovo. Il quale, proprio perché si trattava di un'azione da lui stesso avallata, non si mosse per fermare lo scempio, pur avendone la possibilità e la forza.

Un ulteriore elemento che rende chiaro il modus operandi adottato da Cirillo è la impunità assicurata al «Lector Petrus». Lo rileva opportunamente Diderot - sulla scorta della Historia critica di Brucker - nell'ampia voce Éclectisme dell'Encyclopédie, dove parla di Ipazia e di Cirillo: «M. Brucker oppose à l'innocence du patriarche des présomptions assez fortes; telles que le bruit public ${ }^{22}$, le caractère impétueux de l'homme, le rôle turbulent qu'il a fait de son temps, la canonisation du moine de Nitrie, et l'impunité du lecteur Pierre.»

L'articolo di Diderot fu attaccato dall'abate Claude-Pierre Goujet (1697-1767) nella scia dell'apologetica trattazione di Pierre-Nicolas Desmolets, Dissertation sur

20 Ben lo comprese R. Hoche, Hypatia, Philologus 15 (1860), p. 462.

21 Histoire de la littérature grecque, vol. V, De Boccard, Paris 1928, p. 1028.

22 Si noti il valore di «bruit» che non è affatto termine deteriore. Se n'è parlato supra a proposito della nota di Duchesne stravolta da Fliche e Martin. 
Hypatie, où l'on justifie Saint Cyrille d'Alexandrie sur la mort de cette Sçavante (tomo $\mathrm{V}$ dei Mémoires de littérature et d'histoire). L'intervento di Goujet è nel tomo VI degli stessi Mémoires. Voltaire ironizzò su questa mobilitazione, e nella sua voce Hypatie dell'Encyclopédie scrisse: «Encore une fois deux tomes contre deux pages. Deux lignes contre deux tomes, voilà ce qu'il faut ${ }^{23}{ }_{\text {». }}$

7. Anche se i cattolici sono i più accaniti nella difesa di Cirillo, giova dare uno sguardo anche agli altri. Già si è detto di Jaeger. Un cenno lo merita anche il versante anglicano. Qui ci riferiremo all' Historia litteraria scriptorum ecclesiasticorum di Cave e all'opera di consultazione più recente, l'Oxford Dictionary of the Christian Church, più volte riedito (nel 1997 in terza edizione), pensato e redatto dal Reverendo Frank Lesile Cross (1900-1968), ispiratore nel 1941 della Theological Literature Association nonché a lungo consigliere teologico del vescovo anglicano di Bradford.

Cave se la cava in modo quasi comico: che Ipazia - egli concede - sia stata massacrata dagli Alessandrini è indiscutibile. "Cyrillo autem satagente id evenisse, spectata illius probitas credere nequaquam sinit» (Historia lit., ed. Ginevra 1694, p. 215).

Nell' Oxford Dictionary sia Ipazia che Cirillo ottengono una breve trattazione. Ipazia viene omaggiata come "the glory of the Neoplatonic School of Alexandria», ma, per la questione più delicata, l'impostazione è la medesima del Dizionario ecclesiastico di Mercati e Pelzer ( «la colpevolezza di Cirillo non fu mai provata»), con una inattesa variante onde Oreste da cristiano non fanatico diventa pagano: "On the suspicion that she [Ipazia] had set the pagan [sic] prefect of Alexandria against the Christians, she was attacked by a Christian Mob under Peter the Reader and put to death. The archbishop Cyril was suspected of complicity, but his responsibility was never proveds (p. 813). Curiosamente però la voce Cyril, St. (p. 443), non solo ammette una responsabilità «morale» di Cirillo nell'uccisione di Ipazia, ma mostra schietta ammirazione per la vigorosa azione repressiva del vescovo di Alessandria: «In 412 he succeeded his uncle Theophilus in the patriarchal see. He soon opened warfare with impartial vigour [sic] upon Novatianism, Neoplatonism, the Jews and the imperial prefect Orestes»; quindi prosegue: «And if he himself bore no personal responsibility of the death of the distinguished philosopher Hypatia, her murder was certainly the work of his supporters.»

Luciano CANFORA

Università degli studi di Bari

Piazza Umberto I, 1

I-70121 Bari - Italia

l.canfora@lettere.uniba.it

23 La voce è ristampata nell'edizione ampliata del Dictionnaire philosophique autorizzata dall'autore. (Si veda, nell'edizione parigina del 1821, il t. V, p. 513). 